\title{
Evaluación de plaguicidas para el manejo de plagas del café Coffea arabica L. en Jinotega, Nicaragua
}

\section{Pesticide evaluation for the management of coffee pests Coffea arabica L. in Jinotega, Nicaragua}

\author{
María Nohelia Matus Miranda ${ }^{1}$, Edgardo Jiménez-Martínez ${ }^{2}$ \\ ${ }^{1}$ Master en Sanidad Vegetal, graduada en la Universidad Nacional Agraria \\ ${ }^{2} \mathrm{PhD}$. en Entomología, ORCID: https://orcid.org/0000-0003-1086-7380 / edgardo.jimenez@ci.una.edu.ni / Universidad
} Nacional Agraria, (505) 2263-2609

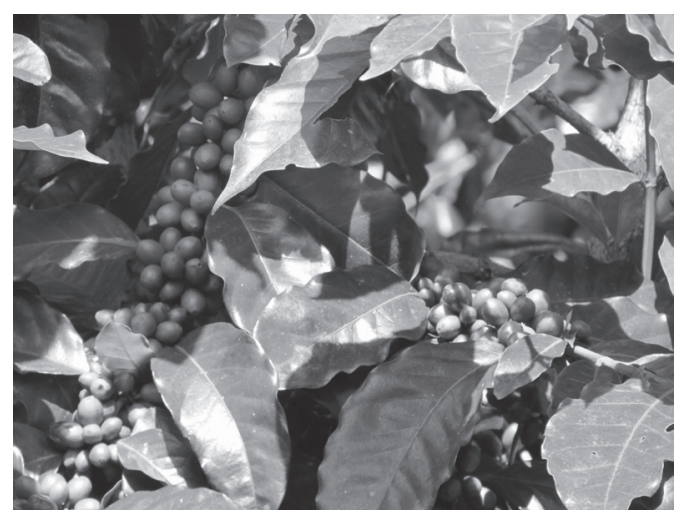

\section{RESUMEN}

El café (Coffea arabica L.) es de mucha importancia para Nicaragua, tanto económica, como social y ambiental, representando aproximadamente el $25 \%$ de las exportaciones. La caficultura es considerada una de las principales fuentes de empleo, alcanzando $63 \%$ en las zonas rurales y alrededor del $14 \%$ a nivel nacional. El cultivo de café es vulnerable a plagas artrópodas y agentes microbiológicos que afectan hojas, tallos, raíces y frutos, causando pérdidas en los rendimientos. La broca del fruto del café (Hypothenemus hampei, Ferrari) (Coleoptera: Curculionidae) es el principal insecto plaga del café en el país por su daño causado y los elevados costos económicos destinados en su manejo. Siguiendo en importancia, la cochinilla (Planococcus spp) (Hemiptera: Pseudococcidae), que infesta brotes foliares y florales, en infestaciones severas, causa amarillamiento de hojas, marchitamiento de yemas y aborto de frutos. Finalmente, las escamas (Coccus viridis, Green) (Hemiptera: Coccidae), insectos con amplio rango de distribución y abundantes en época seca, causan pérdidas por vigor, enanismo y reducción del número de frutos por planta. Este estudio, tuvo el propósito de contribuir al manejo de plagas del café a través de la evaluación de insecticidas químicos, botánicos y biológicos. Las variables evaluadas fueron, número de frutos brocados y número de escamas y cochinillas por planta. Los resultados indican que los mejores insecticidas para el manejo de broca del café, escamas y cochinillas fueron Galil seguido de Cormoran y Ecobiol, los cuales fueron, además, los tratamientos más rentables.

Palabras clave: hypothenemus, cocoidea, escamas, cochinillas, pesticidas.

\begin{abstract}
Coffee (Coffea arabica L.) is of great importance to Nicaragua in aspect of economically, socially and environmentally, representing approximately $25 \%$ of exports. Coffee growing is considered one of the main sources of employment, reaching $63 \%$ in rural areas and around $14 \%$ nationwide. Coffee cultivation is vulnerable to arthropod pests and microbiological agents that affect leaves, stems, roots and fruits, causing losses in yields. The coffee fruit borer (Hypothenemus hampei, Ferrari) (Coleoptera: Curculionidae) is the main coffee pest insect in the country due to damage to the coffee fruits and high cost due to its management. Following in importance, the mealybug (Planococcus spp) (Hemiptera: Pseudococcidae), which infests leaf and flower buds, in severe infestations, causes leaf yellowing, bud wilting and fruit abortion. Finally, the scales (Coccus viridis, Green) (Hemiptera: Coccidae), insects with a wide range of distribution and abundant in the dry season, cause losses due to vigor, dwarfism and a reduction in the number of fruits per plant. This study had the purpose of contributing to the management of coffee pests through the evaluation of chemical, botanical and biological insecticides. The evaluated variables in this study were: number of brocade fruits and number of scales and mealybugs per plant. The results indicate that the best insecticides for the management of coffee bit, scales and mealybugs were Galil followed by Cormoran and Ecobiol, which were also the most profitable treatments.
\end{abstract}

Keywords: Hypothenemus, cocoidea, scales, mealybugs insects, pesticides.
Recibido: 23 de octubre del 2019 Aceptado: 12 de marzo del 2020

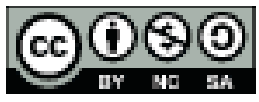

Los artículos de la revista La Calera de la Universidad Nacional Agraria, Nicaragua, se comparten bajo términos de la licencia Creative Commons: Reconocimiento, No Comercial, Compartir Igual. Las autorizaciones adicionales a las aquí delimitadas se pueden obtener en el correo edgardo.jimenez@ci.una.edu.ni 


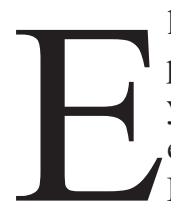
1 café Coffea arabica L. es de mucha importancia para Nicaragua, tanto económica, como social y ambiental, representando aproximadamente el 25\% de las exportaciones (CETREX, 2015). La producción de café es considerada una de las principales fuentes de empleo alcanzando hasta el $63 \%$ en las zonas rurales y alrededor del $14 \%$ en todo el país (MAGFOR, 2013). El cultivo de café es uno de los principales generadores de divisas, alcanzando en el 2015 USD 395.73 millones lo que equivale al $15.6 \%$ del total de exportaciones (CETREX, 2015).

Las exportaciones nicaragüenses en los años 2010-2015 mostraron una tasa media de crecimiento anual de $4.35 \%$ en volumen exportado y un decrecimiento en el valor percibido del $1.38 \%$, producto de los bajos precios internacionales del café en el periodo (OIC, 2016).

La caficultura en Nicaragua se estableció por primera vez en la región del pacífico, específicamente en el departamento de Carazo, para luego dirigirse al centro y norte del país. Actualmente los departamentos de Jinotega y Matagalpa constituyen la zona con mayor área plantada de café, seguida de Nueva Segovia, Carazo y Managua (Kuhl, 2004).

El cultivo de café es vulnerable a las plagas (atrópodos y fitopatógenos) debido que afectan hojas, tallos raíces y frutos. Entre los artrópodos plaga que atacan las raíces se encuentran las palomillas, escamas y nematodos; en tallo y ramas cortadores y taladradores; en hojas cortadores y chupadores; en frutos por la broca; entre los fitopatógenos hongos y con menor frecuencia, bacterias y virus (Guharay, Monterrey, Monterroso y Staver, 2000).

La broca del café Hypothenemus hampei Ferrari (Coleoptera: Curculionidae) es la plaga más destructiva en todos los países productores donde se ha introducido. Este insecto es originario de África Central y fue descrito inicialmente por Ferrari en el año de 1867 (Baker, 1999). En Nicaragua, la presencia de broca del café fue reportada por primera vez en febrero de 1988 en la finca La Fundadora, Matagalpa (MIDINRA, 1988).

El daño causado por este insecto lo inician las hembras adultas, perforando las cerezas por el ombligo, hasta llegar a la almendra, donde se alimentan y adelantan su proceso reproductivo; este daño es característico y consiste en un orificio circular que lo hace en la punta de la fruta, donde hace un túnel para depositar los huevos (Jiménez y Rodríguez, 2014).

Actualmente en la agricultura se hace hincapié en el uso de productos más amigables con el ambiente y la salud humana, aplicando el Manejo Integrado de la Broca (MIB) con métodos de control de tipo manual, usando trampas y manejo biológicos para mantener la broca de la cereza del café en niveles que no ocasionen perjuicio económico (Williamson y Leheup, 2012).
Alvarado y Monroig (2007) mencionan que las cochinillas del café (Planococcus spp) (Hemiptera: Pseudococcidae) conocidas como cochinilla harinosa, son suaves, de color blanco, segregan seda y polvo blanquecino, infestan los brotes tiernos y las yemas florales. Tanto las ninfas como los adultos, chupan la savia de las plantas insertando su estilete en el tejido vegetal. Cuando las infestaciones son severas las hojas se tornan amarillas, las yemas se marchitan y abortan los frutos.

Las escamas del café (Coccus viridis Green) (Hemiptera: Coccidae) se conocen también como conchas, son insectos que tienen un amplio rango de distribución, son abundantes en época de sequía, retardan el crecimiento de la planta al preferir alimentarse de hojas tiernas y terminales jóvenes de los tallos; altas poblaciones causan pérdidas por vigor, enanismos y reducción del número de cerezas por planta (ANACAFE, 2013).

Esta investigación fue motivada por la relevancia que representa en la caficultura y la prohibición del uso de Endosulfán, lo que está ocasionando a los productores el incremento en sus costos de producción y de todas formas, disminución del rendimiento del cultivo; por lo expuesto en esta investigación se estudiaron insecticidas químicos, botánicos y biológicos, para el manejo de la broca y otros insectos plagas asociados al cultivo de café, tomando en consideración que la identificación temprana y el monitoreo constante de plagas, son las principales herramientas a la hora de tomar decisiones de manejo (Jiménez, 2009).

El estudio aspira poner a disposición de los productores caficultores al menos una alternativa, que les permita reducir las poblaciones de plagas a niveles de convivencia, en niveles que no ocasionen pérdidas económicas y disminuir sus costos de producción.

\section{MATERIALES Y MÉTODOS}

Ubicación del área de estudio. El presente estudio se realizó en el período de junio a octubre 2014, en el municipio de San Esteban del departamento de Jinotega, Nicaragua, en la finca agropecuaria Los Potreríos, con un área total de 350 ha; propiedad de los señores: Iván Rizo López y Edwin Rizo López.

El lote evaluado corresponde al nombre de Buena vista, con plantas de Coffea arabica L., var. Catuaí Rojo de 17 años, sembradas a 1.25 metros entre plantas y 2.00 metros entre surcos, al cual se le realizó recepo selectivo en el año 2013, café bajo sombra, provisto por árboles de guaba (Inga vera Willd) a un distanciamiento de $8 \mathrm{~m} \mathrm{x} 12 \mathrm{~m}$.

Material de café evaluado. La variedad de café (Catuaí) utilizada en el estudio, se originó en el Instituto Agronómico de Campinas (IAC), São Paulo, Brasil, como producto del cruce entre Caturra amarillo y Mundo novo, orientado a mejorar la 
productividad, reducir altura de planta $(2.25 \mathrm{~m})$ y aumentar poblaciones por unidad de área.

El Catuaí rojo es de tamaño intermedio con bandolas largas y entrenudos cortos, con hojas terminales de color verde tierno. Los rangos de altitud para su cultivo son desde 600 hasta $1200 \mathrm{msnm}$, observando mejores resultados entre 700 y $1000 \mathrm{msnm}$, variedad resistente al viento y de excelente producción (Hernández, 2012).

Diseño experimental. El ensayo se estableció como un experimento en diseño de bloques completos al azar (BCA), con cuatro repeticiones y seis tratamientos, consistentes de uno botánico, cuatro químicos y un biológico para el manejo de broca, cochinilla y escamas del café.

Tamaño de las parcelas experimentales. Se utilizaron parcelas de seis surcos por $10 \mathrm{~m}$ de largo por dos metros de ancho, para un total de $120 \mathrm{~m}^{2}$ por parcela experimental, con 60 plantas de café.

Tamaño de la parcela útil. La parcela útil consistió en 10 plantas de café tomadas de los dos surcos centrales. De cada planta se tomó una bandola productiva al azar, del estrato superior e inferior, la cual se marcó con cinta plástica de color rojo. El muestreo se realizó cada 15 días utilizando la guía de recuento integral de plagas (Alvarado y Moroig, 2007), la cual fue modificada según datos encontrados. El área de cada bloque fue de $720 \mathrm{~m}^{2} \mathrm{y}$ el área total de $2880 \mathrm{~m}^{2}$.

Identificación de insectos plagas del café. $\mathrm{La}$ identificación de los insectos plagas del café se hizo al nivel de orden, familia y género, utilizando para la identificación morfológica de los especímenes, un estereoscopio CARL ZEISS (modelo 475002 y 475002 - 9902 de 4x, 6.3x y $2.5 x$ ). La identificación al nivel de familia se realizó en el laboratorio de entomología de la Universidad Nacional Agraria, utilizando las claves taxonómicas dicotómicas propuestas por Nunes y Dávila (2004).

La identificación hasta el taxón de género, a fin de corroborar lo que consta en documentos científicos, se hizo llevando los especímenes al técnico Alex Armando Cerrato del museo entomológico de la UNA, quien, con la ayuda de especímenes de referencia del museo, hizo comparaciones y los pasó por claves dicotómicas morfológicas según la literatura de Andrews y Caballero 1989; Jiménez-Martínez 2009; Maes, 1998; y Sáenz y De la Llana, 1990.

Muestreo de insectos plagas del café. La metodología utilizada fue la propuesta por Guharay et al. 2000, tomando como referencia la hoja de recuento integral de plagas en campo, seleccionando los puntos para cada bloque y tratamientos, determinando el momento de la aplicación de los tratamientos con base en muestreos quincenales durante la mañana. Para esto se seleccionaron 10 plantas entre los dos surcos centrales de cada parcela, revisando las bandolas en el haz y envés, de los estratos superior e inferior, anotando los datos obtenidos de cada variable. Con base a lo recomendado por V. Aguilar (Comunicación personal 2014), quince días antes de la primera aplicación se realizó un muestreo por la mañana, seleccionando los dos surcos centrales de cada parcela y revisando 10 plantas. Por cada planta se revisaron las bandolas, nudos, hojas, frutos y yemas terminales, por el haz y el envés; el número de insectos encontrados por punto de muestreo se anotaron en la hoja de recuento integral que fue modificada según datos obtenidos en campo.

\section{Tratamientos evaluados}

Tratamiento 1: BRALIC 12.5 EC. SegúnADAMA(2018a), este insecticida es un repelente botánico con base en extracto esencial de ajo, el cual es absorbido por el sistema vascular de la planta, alterando el sistema enzimático, provocando cambios en la transpiración mediante la modificación de los jugos intracelulares. Enmascara el efecto de las feromonas producidas por los insectos, rompiendo el ciclo de vida de estos, desvía los hábitos alimenticios de los insectos y ataca su sistema nervioso central mediante sustancias azufradas llamadas alomonas.

Tratamiento 2: GRIZLY 22.5 SC. Según ADAMA (2018b), es un insecticida con base en Imidacloprid, Novaluron y Bifentrina. Actúa en forma sistémica, por ingestión y por contacto. Este insecticida combina tres ingredientes activos: Imidacloprid, insecticida de acción sistémica, actúa por contacto e ingestión, pertenece al grupo de los neonicotinoides que actúan sobre el sistema nervioso central de los insectos; el Novaluron actúa principalmente por ingestión, tiene un alto porcentaje de mortalidad en los primeros estadios larvales (L1 - L2), es supresor de la fecundidad; y, la Bifentrina, que actúa por contacto e ingestión interfiriendo en la membrana nerviosa, la dosis recomendada es de $400 \mathrm{ml}$ en 200 litros de agua por hectárea. La dosis utilizada en este tratamiento en el ensayo fue de $64 \mathrm{ml}$ por bomba de 20 litros.

Tratamiento 3: GALIL 30 SC. Según ADAMA (2018c), es un insecticida con base en Imidacloprid 25\% (Neonicotinoide, sistémico y de contacto) y Bifentrina 5\% (Piretroide de contacto e ingestión). El Imidacloprid pertenece al grupo de los neonicotinoides, actúa sobre el sistema nervioso central de los insectos, causando un bloqueo irreversible de los receptores de la acetilcolina. La Bifentrina interfiere en la membrana de la célula nerviosa, la dosis recomendada es de $400 \mathrm{ml}$ en 200 litros de agua por hectárea. La dosis utilizada en este tratamiento fue de $64 \mathrm{ml}$ por bomba de 20 litros.

Tratamiento 4: ECOBIOL 5 PM (Beauveria bassiana). Monzón (2004), describe que B. bassiana es un hongo 
imperfecto de la clase Deuteromycetes, capaz de infectar a más de 200 especies de insectos. Es de apariencia polvosa, de color blanco algodonoso o amarillento cremoso. El ciclo de vida de este hongo consta de dos fases: la patogénica y la saprofítica. El desarrollo del hongo se puede dividir hasta en ocho etapas. Beauveria bassiana debe aplicarse bajo condiciones propicias para su desarrollo, deben prevalecer condiciones ambientales idóneas (temperatura y humedad) y la presencia de hospederos (plaga objetivo). La forma de aplicación más común es la foliar, empleando formulaciones líquidas (pH 6 o 7), o sólidas.

Tratamiento 5: CORMORAN 18 EC. Según ADAMA (2018d), es un insecticida que contiene los ingredientes activos Novaluron y Acetamiprid, que proporciona un nuevo modo de acción con rápido efecto de volteo y un control prolongado sobre insectos masticadores y chupadores. Cormoran, es una mezcla de manejo antirresistencia, con acción ovicida, de contacto, ingestión, sistémico y translaminar. Novaluron pertenece al grupo de las benzoilfenil ureas, que se caracteriza por inhibir la formación de quitina y producir la muerte de los insectos al momento de la muda (evita que los insectos se conviertan en adultos). Desde que el insecto toma contacto con el producto, disminuye el daño (deja de comer) pudiendo transcurrir dos o tres días hasta que se produce la muerte. Es un larvicida que actúa por ingestión. También actúa por contacto como ovicida y supresor de la fecundidad en hembras adultas. Acetamiprid es un insecticida de la familia de los neonicotinoides, sistémico y translaminar, muy bien absorbido por el follaje; con acción rápida y de efecto residual prolongado (no se lava); actúa sobre los organismos nocivos por ingestión y por contacto. Tiene un mecanismo de acción sobre el sistema nervioso central de los insectos, interfiriendo con la neurotransmisión en las plagas objetivo.

Tratamiento 6: RIMON 10 EC. Según ADAMA (2018e), es un insecticida que pertenece al grupo de las benzoilfenil ureas, que se caracteriza por inhibir la formación de quitina (evita que los insectos se conviertan en adultos). Desde que el insecto toma contacto con el producto disminuye el daño (deja de comer) pudiendo transcurrir dos o tres días hasta que se produzca la muerte. Es un larvicida que actúa por ingestión. También actúa por contacto como ovicida y supresor de la fecundidad en hembras adultas. Provoca la muerte de las larvas en sus primeros estadios (L1 y L2) causando la muerte de uno a tres días posteriores a la aplicación.

\section{Variables evaluadas}

Número de frutos brocados por planta. Esta variable se registró 50 días antes de realizar la primera aplicación, los frutos se contaron y se revisaron principalmente en la corona, lugar donde se encuentra más frecuentemente la broca.
Fluctuación poblacional de escamas (Coccus viridis). Esta variable se tomó 50 días antes de realizar la primera aplicación, revisando y muestreando la bandola en su totalidad principalmente en el punto de crecimiento apical, nudos y frutos, lugares donde se encuentran más frecuentemente las escamas. Posteriormente se realizaron recuentos cada 15 días.

Fluctuación poblacional de cochinillas (Planococcus spp.). Esta variable se tomó 50 días antes de realizar la primera aplicación, revisando y muestreando la bandola en su totalidad principalmente en el punto de crecimiento apical, nudos y frutos, lugares donde se encuentran más frecuentemente las cochinillas. Posteriormente se realizaron recuentos cada 15 días.

Rendimiento (kg ha ${ }^{-1}$ ). Para obtener los datos de rendimiento por hectárea se tomó el peso de frutos maduros de 10 plantas de cada tratamiento, en libras, y posteriormente se transformó a $\mathrm{kg} \mathrm{ha}^{-1}$. Esta información se tomó dos meses posterior a las aplicaciones de insecticidas.

Análisis económico de los rendimientos. Los resultados que se obtuvieron del experimento de campo fueron sometidos a un análisis económico, con el propósito de determinar la rentabilidad de los tratamientos en comparación con la práctica común de los productores, así como el tratamiento con mejor retorno económico. Todo tratamiento recomendado en la producción debía ajustarse a los objetivos y circunstancias de los productores.

Se tomaron los datos de rendimiento promedio $(\mathrm{R} \chi)$ por tratamiento y se obtuvo el rendimiento ajustado (Rajust $=10 \%$ de $\mathrm{R} \chi$ ). Luego se calculó el beneficio bruto multiplicando el Rajust por el precio de venta de campo 2.77 USD kg-1. Para la sumatoria de los costos totales que varían, se estimó los costos de los insecticidas evaluados más el costo de aplicación. Para obtener los costos fijos se incluyó la depreciación de equipos usados, costos de insumos usados, mano de obra, control de plagas, etc. Al obtener el beneficio neto, se restó los costos variables menos los costos fijos de cada tratamiento (CIMMYT, 1988).

Costos variables por parcela. Son todos aquellos costos por unidad de área relacionados con los insumos comprados, labores mecánicas, maquinaria, etc., que varían de un tratamiento a otro.

Costos totales por parcela. Es la suma de los costos fijos y los costos variables.

Rendimiento bruto. La producción de cada uno de los tratamientos por unidad área. 
Rendimiento ajustado. Es el rendimiento bruto reducido en un determinado porcentaje, con el propósito de reflejar la diferencia entre el rendimiento experimental y el que podría ser obtenido por el productor con ese mismo tratamiento.

Precio del producto. Es la relación de cambio por dinero, se refiere al número de unidades monetarias que se necesitan para obtener a cambio una unidad de producto.

Análisis estadístico de los datos. Se efectuó la recolección de los datos de campo y se ordenaron por variables. A cada variable en estudio se les realizó un análisis de varianza (ANDEVA) y luego una comparación por medio de la prueba de separación de medidas de TUKEY al 5\%, mediante el programa estadístico Infostat 2005. Los datos se transformaron con la raíz cuadrada de $(\mathrm{y}+0.5)$. Se realizó una comparación de los rendimientos en $\mathrm{kg} \mathrm{ha}^{-1}$ de los tratamientos evaluados, sometiendo los datos a un análisis económico de presupuesto parcial, análisis de dominancia y análisis de la tasa de retorno marginal.

\section{RESULTADOS Y DISCUSIÓN}

Número de frutos brocados por planta. se realizó comparación de los daños de frutos de café hechos por la Broca en el período de junio a octubre del 2014 (Figura 1). En todos los tratamientos y las fechas de muestreo se presentaron daños de éste insecto, el mayor daño de frutos por broca fue encontrado en la fecha octubre 6 con 0.71 insectos por planta en el tratamiento Rimon, seguido de agosto 14 con 0.55 frutos brocados por planta en el tratamiento Grizly. El análisis de varianza de la variable frutos brocados por planta, mostró diferencia significativa entre los tratamientos $(p=0.0407)$, donde el menor número de frutos dañados por $H$. hampei fue encontrado en el tratamiento Galil, con un promedio de 0.02 frutos brocados por planta, seguido del tratamiento Cormoran, con un promedio de 0.05 frutos brocados por planta y Ecobiol, con un promedio de 0.09 (Cuadro 1).

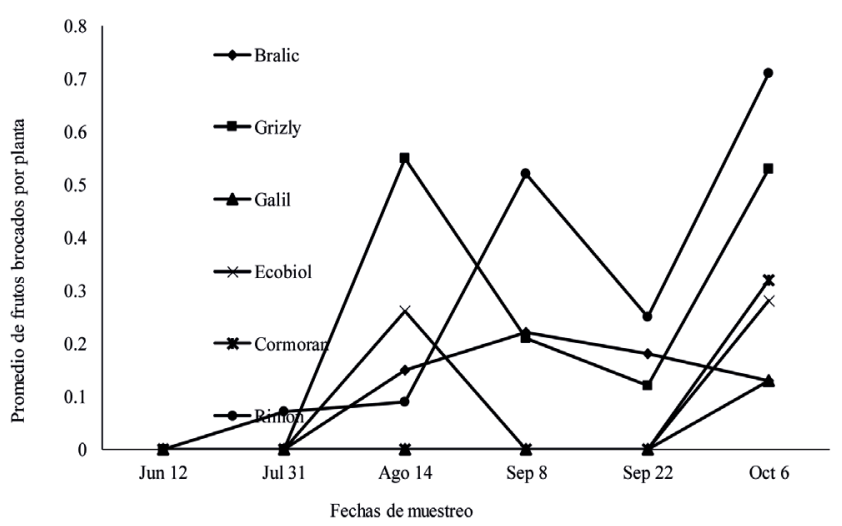

Figura 1. Frutos de café dañados por broca del café según tratamiento y fechas de muestreo.
Cuadro 1. Frutos dañados por broca según tratamiento

\begin{tabular}{lc}
\hline & $\begin{array}{c}\text { Promedio de frutos brocados } \\
\text { por planta } \\
\text { Tratamiento }\end{array}$ \\
\hline Galil & $0.02 \pm 0.02 \mathrm{a}$ \\
Cormoran & $0.05 \pm 0.04 \mathrm{a}$ \\
Ecobiol & $0.09 \pm 005 \mathrm{ab}$ \\
Bralic & $0.11 \pm 0.05 \mathrm{ab}$ \\
Grizly & $0.23 \pm 0.08 \mathrm{~b}$ \\
Rimon & $0.27 \pm 0.10 \mathrm{~b}$ \\
\hline $\mathrm{N}$ & 144 \\
$\mathrm{CV}(\%)$ & 212.87 \\
(F; gl; $p$ ) & $(2.39 ; 138 ; 0.0407)$
\end{tabular}

Tukey (alfa: 0.05), ES: Error Estándar, CV: Coeficiente de Variación,

$\mathrm{N}$ : Número de datos utilizados en el análisis, F: Fisher calculado, gl: grados de libertad del error, $p$ : Probabilidad según Tukey.

Fluctuación poblacional de escamas (Coccus viridis). Estos hemípteros se encontraron en todas las fechas de muestreo (Figura 2), aunque los mayores picos poblacionales se presentaron en agosto 14 , con 4.38 escamas por planta enel tratamiento Bralic y 3.93 escamas por planta en el tratamiento Ecobiol; en tanto que en septiembre 8 se encontraron 3.00 escamas por planta en el tratamiento Ecobiol y en septiembre 22 se encontraron 1.83 escamas por planta en los tratamientos Cormoran y Bralic.

El análisis de varianza realizado a los tratamientos evaluados indica que existe diferencia significativa entre los tratamientos $(p=0.0044)$, donde el menor número de Escamas fue encontrado en el tratamiento Galil con un promedio de 0.1 escamas por planta, seguido del tratamiento Rimon con un promedio de 0.19 escamas por planta y Grizly con un promedio de 0.34 escamas por planta (Cuadro 2).

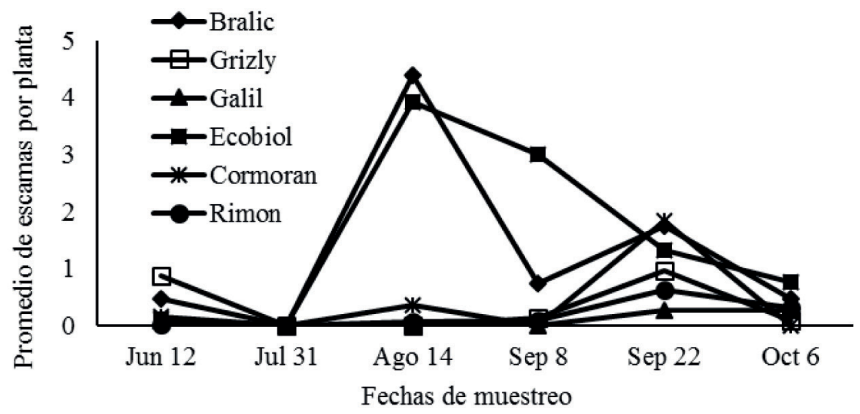

Figura 2. Fluctuación poblacional de escamas Coccus viridis según tratamiento y fechas de muestreo. 
Cuadro 2. Fluctuación poblacional de escamas (Coccus viridis) según tratamiento

\begin{tabular}{lc}
\hline Tratamiento & $\begin{array}{c}\text { Promedio } \\
\text { (Coccus viridis) por planta } \\
\text { Medias } \pm \text { ES }\end{array}$ \\
\hline Galil & $0.10 \pm 0.04 \mathrm{a}$ \\
Rimon & $0.19 \pm 0.08 \mathrm{a}$ \\
Grizly & $0.34 \pm 0.14 \mathrm{ab}$ \\
Cormoran & $0.39 \pm 0.23 \mathrm{ab}$ \\
Bralic & $1.30 \pm 0.52 \mathrm{ab}$ \\
Ecobiol & $1.52 \pm 0.52 \mathrm{~b}$ \\
\hline $\mathrm{N}$ & 144 \\
$\mathrm{CV}$ & 156.35 \\
$(\mathrm{~F} ; \mathrm{gl} ; p)$ & $(3.59,138,0.0044)$ \\
\hline
\end{tabular}

Tukey(alfa: 0.05),ES:ErrorEstándar, CV:Coeficiente de variación, $\mathrm{N}$ : Número de datos utilizados en el análisis, F: Fisher calculado, gl: grados de libertad del error, p: Probabilidad según Tukey.

Fluctuación poblacional de cochinillas Planococcus spp. $\mathrm{El}$ análisis de varianza mostró diferencia significativa $(\mathrm{p}=$ 0.0493) entre los tratamientos (Cuadro 3); donde el menor número de cochinillas fue encontrado en el tratamiento Galil, con un promedio de 0.02 cochinillas por planta, seguido del tratamiento Grizly, con un promedio de 0.09 cochinillas por planta y Cormoran, con un promedio de 0.17 cochinillas por planta (Cuadro 3).

Cuadro 3. Fluctuación poblacional de cochinilla Planococcus spp

\begin{tabular}{cc}
\hline & $\begin{array}{c}\text { Promedio de } \begin{array}{c}\text { Planococcus spp por } \\
\text { planta } \\
\text { Medias } \pm \mathrm{ES}\end{array} \\
\text { Tratamiento }\end{array}$ \\
\hline Galil & $0.02 \pm 0.01 \mathrm{a}$ \\
Grizly & $0.17 \pm 0.05 \mathrm{a}$ \\
Cormoran & $0.10 \pm 0.08 \mathrm{ab}$ \\
Rimon & $0.27 \pm 0.10 \mathrm{~b}$ \\
Ecobiol & $0.33 \pm 0.14 \mathrm{~b}$ \\
Bralic & 144 \\
$\mathrm{~N}$ & 175.77 \\
$\mathrm{CV}$ & $(2.02 ; 138 ; 0.0493)$ \\
\hline F $;$ gl; P $)$
\end{tabular}

Tukey (alfa: 0.05), ES: Error Estándar, CV: Coeficiente de Variación, $\mathrm{N}$ : Número de datos utilizados en el análisis, F: Fisher calculado, gl: grados de libertad del error, P: Probabilidad según Tukey.

Los sistemas productivos de café en Nicaragua, se caracterizan por ser sistemas de producción que tratan de utilizar al máximo los recursos de la finca, dándole énfasis a la fertilidad del suelo, usando fertilizantes orgánicos o químicos y plaguicidas tanto naturales como sintéticos para el manejo del cultivo y sus plagas (Pereira y Parrales, 2006).
De acuerdo a los resultados obtenidos en este estudio, los mejores insecticidas para la supresión (manejo) de las poblaciones de la broca del café fueron Galil y Grizli.

Vinces (2018) usó Cormoran ${ }^{\circledR}$ para el control del pulgón negro de los cítricos (Toxoptera aurantii, Boyer de Fonscolombe), en limonero (Citrus aurantifolia, Swingle) en Perú, resultando ser efectivo contra esta plaga sin presentar efectos negativos sobre la fauna benéfica. Mejia (2016) evaluó la eficacia biológica de plaguicidas entre ellos Cormoran ${ }^{\circledR}$ sobre el insecto Diaphorina citri Kuwayama (Hemiptera: Liviidae) a las 2, 24, 48, 72 y 144 horas, in vitro, en brotes y plántulas, obteniendo efectividad sobre estos insectos en brotes y plántulas desde 93 al 100\%.

El insecticida biológico Ecobiol con base en B. bassiana, tuvo un buen desempeño en este estudio, aunque por si solo no es suficiente contra la broca del café, obteniendose un promedio de 0.09 frutos brocados por planta en este estudio. Sin embargo, actualmente se sabe que el uso de estos agentes de control biológico integrados con la destrucción de frutos infestados, estrategia que ha sido denominada Manejo Integrado de la Broca (MIB), ofrecen una importante reducción en los porcentajes de infestación en campo (Guharay et al., 2000).

Aunque $B$. bassiana es un hongo que actúa desde el inicio del tratamiento, su efectividad se observa a partir del cuarto día. Este hongo ha sido aislado de más de 200 especies de insectos de diferentes órdenes, incluyendo plagas de cultivos de importancia económica (Alves, 1998). $B$. bassiana actúa por contacto en los diferentes estadios de los insectos plaga.

Las conidias, son las unidades infectivas, penetran al cuerpo del insecto, produciéndole disturbios al nivel digestivo, nervioso, muscular, respiratorio, excretorio, etc; es decir el insecto se enferma, deja de alimentarse y posteriormente muere. La muerte puede ocurrir entre los tres a cinco días, dependiendo de la virulencia del hongo y estadio del insecto (Monzón, 2004). Bustillo, Bernal, Benavides y Chavez (1999) reportaron una eficiencia de control del $30 \%$ de B. basiana sobre H. ampei en 350 frutos brocados el mismo día de la aspersión y 2, 5, 10, 15, 20, 25 y 30 días después de la aspersión.

De La Rosa, Alatorre, Barrera y Toriello (2000) investigarón el efecto de tres cepas del hongo B. bassiana sobre el barrenador de café, $\mathrm{H}$. hampei en tres fincas de café a diferentes altitudes $(450-1100 \mathrm{~m}$ sobre el nivel del mar) en Soconusco, Chiapas, México. Ellos encontraron que el porcentaje medio máximo de micosis varía según la altitud. A $450 \mathrm{msnm}$ la micosis fue de $14.30 \%$, a $880 \mathrm{msnm}$ la micosis fue del $40.60 \%$ y a $1100 \mathrm{msnm}$ fue de $33.90 \%$.

Siu (2018), investigó el uso de Beauveria bassiana (Bals y Vuils), equipos de aplicación y prácticas culturales para el manejo de broca del café (Hypothenemus hampei, Ferrari), en el cultivo del café (Coffea arabica L.) en Jalapa, 
Nueva Segovia, 2016-2017. Encontrando que la menor incidencia de broca en el campo se registró en el programa de manejo que incluyó pepena, trampa y $B$. bassiana aplicado al suelo y a la planta con bomba de motor y el menor porcentaje de daño en café pergamino se encontró en café proveniente de parcelas donde se realizó pepena y $B$. bassiana aplicada a la planta con bomba de motor.

Monzón (2004), investigó las formulaciones de Beauveria bassiana (Bals y Vuils) para el manejo de plagas en el cultivo del repollo (Brassica oleracea L. var capitata) en el Tisey,

Estelí. Encontrando que la concentración de conidias de B. bassiana en todas las formulaciones se mantuvo estable durante seis meses en todas las formulaciones. La viabilidad de conidias de $B$. bassiana en todas las formulaciones se mantuvo estable, con $97 \%$ a los tres meses y disminuyendo a $82 \%$ a los seis meses y que el efecto de las formulaciones sobre las plagas de repollo fue variable. $B$. bassiana en aceite tuvo el mejor efecto sobre Plutella xylostella, Leptophobia sp. y áfidos; en cambio el mejor efecto sobre Diabrotica sp. se registró en la formulación con arcilla blanca.

\section{Comparación económica de los tratamientos evaluados}

Presupuesto parcial. El análisis del presupuesto parcial realizado según la metodología del CIMMYT, 1988 se determinó que los mayores costos variables los obtuvieron los tratamientos Grisly, Galil y Bralic con 30.08, 27.52 y 24.48 USD ha $^{-1}$, los de menor costos variables fueron los tratamientos Rimon, Cormoran y Ecobiol con 24.40, 23.70 y 20.32 USD ha $^{-1}$ respectivamente.

El tratamiento que obtuvo el mayor beneficio neto fue Galil con 984.17 USD ha ${ }^{-1}$, en cambio el tratamiento que presentó los menores beneficios netos fue Rimon con 273.21 USD ha-1 (Cuadro 3).

Control botánico USD ha ${ }^{-1}$ (Depende del \# de bombadas y costo del tratamiento)

Control biológico USD ha ${ }^{-1}$ (Depende del \# de bombadas y costo del tratamiento)

del \# de bombadas y costo del tratamiento)

Costo de aplicación en USD ha ${ }^{-1}$

Depreciación de bomba de mochila/
Los resultados de este experimento fueron sometidos a análisis económico. El propósito fue determinar el tratamiento con mejor retorno económico. Todo tratamiento recomendado en la producción debe ajustarse a los objetivos y circunstancias de los productores, por lo tanto, el proceso de aplicación de este enfoque debe generar una recomendación para los agricultores (CIMMYT, 1988).

El análisis de presupuesto parcial refleja que el tratamiento Ecobiol presentó los menores costos variables y el tratamiento Grisly los mayores costos variables.

Cuadro 4. Presupuesto parcial en dólares (USD) según tratamientos

\begin{tabular}{|c|c|c|c|c|c|c|}
\hline Concepto & Galil & Cormoran & Ecobiol & Bralic & Grizly & Rimon \\
\hline Rendimiento $\left(\mathrm{kg} \mathrm{ha}^{-1}\right)$ & 807.99 & 751.43 & 694.87 & 638.31 & 581.75 & 525.19 \\
\hline Rendimiento ajustado $(10 \%)\left(\mathrm{kg} \mathrm{ha}^{-1}\right)$ & 727.19 & 676.28 & 625.38 & 574.47 & 523.58 & 472.67 \\
\hline Precio de campo (USD kg-1) & 2.77 & 2.77 & 2.77 & 2.77 & 2.77 & 2.77 \\
\hline Ingreso bruto (USD ha-1) & 2014.32 & 1873.32 & 1732.31 & 1591.31 & 1450.30 & 1309.30 \\
\hline
\end{tabular}

8.48 4.32

$\begin{array}{llll}11.52 & 7.70 & 14.08 & 8.40\end{array}$

$\begin{array}{lrrrrr}\text { (Depende del \# de bombadas a aplicar) } \quad 16.00 & 16.00 & 16.00 & 16.00 & 16.00 & 16.00\end{array}$

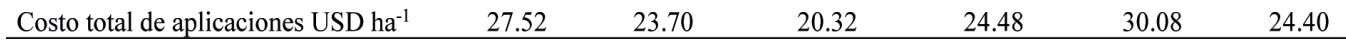
Costos fijos ciclo

Costo de Manejo de Sombra/ciclo Costo de semilla (USD ha-1)

Costo total de MO (USD ha-1)

Costo de fertilizantes, Fungicidas y

otros

otal de CF (USD ha"-1)

$\begin{array}{rrrrrr}7.59 & 7.59 & 7.59 & 7.59 & 7.59 & 7.59 \\ 8.00 & 8.00 & 8.00 & 8.00 & 8.00 & 8.00 \\ 60.00 & 60.00 & 60.00 & 60.00 & 60.00 & 60.00\end{array}$

$\begin{array}{llllll}610.96 & 610.96 & 610.96 & 610.96 & 610.96 & 610.96\end{array}$

$\begin{array}{lrrrrr}325.14 & 325.14 & 325.14 & 325.14 & 325.14 & 325.14\end{array}$

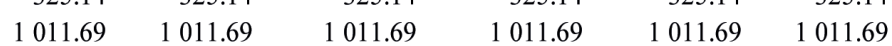

$\begin{array}{llllll}27.52 & 23.70 & 20.32 & 24.48 & 30.08 & 24.40\end{array}$

$\begin{array}{llllll}984.17 & 837.93 & 700.3 & 555.14 & 408.53 & 273.21\end{array}$

** MO: Mano de obra, CV: Costos variables, CF: Costos fijos; ** Precio oficial del dólar: 26.45; ** Precio del producto al momento de la cosecha 2.77 USD kg- ${ }^{1}$.

Los tratamientos Galil y Cormoran fueron los que obtuvieron los mayores beneficios netos, por el contrario, el tratamiento que obtuvo el menor beneficio neto fue Rimon, con 273.21 dólares por hectárea (Cuadro 4).

Análisis de dominancia. Para realizar el análisis de dominancia se tomó en cuenta el análisis de presupuesto parcial, se consideran los costos variables de cada tratamiento y si los costos variables de un tratamiento están por debajo de los costos totales de producción, se considera un tratamiento dominado. 
El resultado del análisis de dominancia indica que los tratamientos Bralic, Grizly y Rimon resultaron ser dominados por los tratamientos Galil, Cormoran y Ecobiol. Por lo tanto, no fueron incluidos en el análisis de la tasa de retorno marginal dado que si son considerados se obtendría una tasa de retorno marginal negativa.

El análisis de dominancia realizado a este estudio refleja que los tratamientos Bralic, Grizly y Rimon resultaron ser dominados, esto se debe a que presentan menores beneficios netos y mayores costos variables que el resto de los tratamientos incluidos en este estudio, por lo tanto, estos fueron excluidos para la realización del análisis de la tasa de retorno marginal. Los tratamientos Galil, Cormoran y Ecobiol resultaron ser no dominados de acuerdo con el análisis de la tasa de retorno marginal (Cuadro 5).

Cuadro 5. Análisis de dominancia

\begin{tabular}{cccc}
\hline Tratamiento & $\begin{array}{c}\text { Costo variable } \\
\left(\text { USD ha }^{-1}\right)\end{array}$ & $\begin{array}{c}\text { Beneficio neto } \\
\left(\text { USD ha }^{-1}\right)\end{array}$ & Dominancia \\
\hline Ecobiol & 20.32 & 700.30 & ND \\
Cormoran & 23.70 & 837.93 & ND \\
Rimon & 24.40 & 273.21 & D \\
Bralic & 24.48 & 555.14 & D \\
Galil & 27.52 & 984.17 & ND \\
Grizly & 30.08 & 408.53 & D \\
\hline
\end{tabular}

**ND: No dominado D: Dominado.

Análisis de la tasa de retorno marginal (TRM). El análisis de la tasa de retorno marginal refleja que para el control de broca del café el mejor tratamiento es el Cormoran, ya que por cada dólar invertido el agricultor obtiene una tasa de retorno marginal de $4.072 \%$, es decir que por cada dólar invertido se recupera dicho dólar y 40.72 dólares adicionales, siendo estos beneficios mayores que los que aportan los otros tratamientos comparados. Si se usa Galil para el control de broca, por cada dólar invertido se obtiene una tasa de retorno marginal de $3.828 \%$, lo cual equivale a 38.28 dólares adicionales una vez recuperado el dólar invertido (Cuadro 6).

Cuadro 6. Análisis de la tasa de retorno marginal según tratamientos no dominados.

\begin{tabular}{cccccc}
\hline Tratamiento & $\begin{array}{c}\text { Costo Variable } \\
\left(\text { USD ha }^{-1}\right)\end{array}$ & $\begin{array}{c}\text { Costo Marginal } \\
\left(\text { USD ha }^{-1}\right)\end{array}$ & $\begin{array}{c}\text { Beneficio Neto } \\
\left(\text { USD ha }^{-1}\right)\end{array}$ & $\begin{array}{c}\text { Beneficio Marginal } \\
\left(\text { USD ha }^{-1}\right)\end{array}$ & $\begin{array}{c}\text { Tasa de Retorno } \\
\text { Marginal } \\
(\%)\end{array}$ \\
\hline Ecobiol & 20.32 & & 700.30 & & \\
Cormoran & 23.70 & 3.38 & 837.93 & 137.63 & 4.072 \\
Galil & 27.52 & 3.82 & 984.17 & 146.24 & 3.828 \\
\hline
\end{tabular}

\section{CONCLUSIONES}

El insecticida Galil, seguido de Cormoran fueron los tratamientos más efectivos para el manejo de plagas del café.

Los tratamientos Galil, seguido de Cormoran y Ecobiol fueron los que obtuvieron los mayores rendimientos en kilogramos por hectárea.

En el análisis de presupuesto parcial, los tratamientos que obtuvieron los mejores beneficios netos fueron Galil, Cormoran y Ecobiol.

El análisis de dominancia realizado en este estudio demuestra que los tratamientos Galil, Cormoran y Ecobiol resultaron no dominados, siendo estos los tratamientos más viables económicamente.

El análisis de la tasa de retorno marginal indica que el tratamiento Cormoran tiene una tasa de retorno marginal de $4.072 \%$, es decir que por cada dólar invertido se obtiene una ganancia de 40.72 dólares adicionales y si usa Galil se obtiene una tasa de retorno de $3.828 \%$ es decir que por cada dólar invertido se obtiene una ganancia de 38.28 dólares netos adicionales.

\section{REFERENCIAS BIBLIOGRÁFICAS}

ADAMA-Essencials. (2018a). Bralic 12.5 EC. https:/www.adama.com/central-america/es/portafolio-deluciones/insecticidas/bralic12.5-ec.

ADAMA-Essencials. (2018b). Grizly 22.5 SC. https:/www.adama.com/central-america/es/portafolio-de-oluciones/insecticidas/grizlymax-22-5-sc.

ADAMA-Essencials. (2018c). Galil-30 SC. https://www.adama.com/central-america/es/portafolio-de-soluciones/insecticidas/galil-30Sc

ADAMA-Essencials. (2018d). Cormoran 18 EC. https://www.adama.com/central-america/es/portafolio-de-Soluciones/insecticidas/ cormoran-18-ec

ADAMA-Essencials. (2018e). Rimon 10 EC. https://www.adama.com/central-america/es/portafolio-de-soluciones/insecticidas/rimon10 -ec.

Alvarado O., y Moroig, M. (2007). Guía práctica de plagas y enfermedades en café, Servicio de extensión agrícola- colegio de ciencias agrícolas. Mayagüez, Puerto Rico: Universidad de Puerto Rico. 
Alves, S. (1998). Controle microbiano de insetos. $2^{\mathrm{a}}$ Ed. Brasil. 74 pp.

Asociación Nacional del Café. (2013). Las escamas foliares del cafeto. https://www.anacafe.org/glifos/index.php?title=Escamas foliares_del_cafeto

Andrews Keith. L., y Caballero, R. (1989). Guia para el estudio de órdenes y familias de insectos de Centroamérica. Francisco Morazán, Honduras: Escuela Agrícola Panamericana El Zamorano.

Baker, P. S. (1999). La broca del cafeto y su manejo en México (Coleóptera, Scolytidae). Entomológica Mexicana 61. pp. 29-41.

Bustillo, A. E., Bernal, M. G., Benavides, P., y Chaves, B. (1999). Dynamics of Beauveria bassiana and Metarhizium anisopliae infecting Hypothenemus hampei (Coleoptera: Scolytidae) populations emerging from fallen coffee berries. Florida Entomologist 82(4). 492498

Centro Internacional de Mejoramiento de Maíz y Trigo. (1988). La formulación de recomendaciones a partir de datos agronómicos: Un manual metodológico de evaluación económico. Distrito Federal, Mexico: CIMMYT.

CETREX (Centro de Trámites para las Exportaciones). (2015). Números de la cosecha de café en Nicaragua. https://www. centralamericadata.com/es/article/home/Nmeros_de_la_cosecha_de_caf_en_Nicaragua.

De La Rosa, W., Alatorre, R., Barrera, J. F., y Toriello, C. (2000). Effect of Beauveria bassiana and Metarhizium anisopliae (Deuteromycetes) upon the Coffee Berry Borer (Coleoptera: Scolytidae) Under Field Conditions. Journal of Economic Entomology, 93 (5). 1409-1414.

Guharay F., Monterrey, J., Monterroso, D., y Staver, C. (2000). Manejo integrado de plagas en el cultivo del café. Managua, Nicaragua: CATIE.

Hernández, O. (2012). El Café y sus variedades. El Salvador. Consultado el 15 feb. 2018. Disponible en http:/hablemosdelcafe.blogspot. com/2012/10/el-cafe-y-sus-variedades.html

Jiménez Martínez, E. S. (2009). Entomología. Managua, Nicaragua: UNA.

Jimenez Martinez, E. S. (2009). Manejo Integrado de plagas. Managua Nicaragua: UNA.

Jiménez Martínez, E. S., y Rodríguez, O. (2014). Insectos plagas de cultivos en Nicaragua. Managua, Nicaragua: UNA.

Kuhl A, E. (2004). Nicaragua y su café. Managua, Nicaragua. Hispamer.

Maes, J. M. (1998). Insectos de Nicaragua: catálogo de los insectos y artrópodos terrestres de Nicaragua. León, Nicaragua.

Mejía, L. M. (2016). Evaluación de eficacia biológica de plaguicidas sobre Diaphorina citri Kuwayama (Hemiptera: Liviidae). Tesis de pregrado. Universidad Nacional de Colombia, $82 \mathrm{pp}$.

MIDINRA (Ministerio de Desarrollo, Industria y Reforma Agraria). (1988). Guía Fitosanitaria para el cultivo del cafeto con énfasis en la Broca del Café (Hypothenemus hampei Ferr). Managua, Nicaragua.

Ministerio Agropecuario y Forestal (2013). El café en Nicaragua. Managua, Nicaragua: MAGFOR.

Monzón, A.J. (2004). Guía para el control biológico de la broca del café Hypothenemus hampei, Guía técnica No. 6. Managua, Nicaragua: UNA.

Nunes Zuffo, C., y Dávila Arce, M. L. (2004). Taxonomia de las Principales Familias y Subfamilias de Insectos de interés Agrícolas en Nicaragua. Estelí, Nicaragua: UCATSE.

OIC (Organización Internacional del Café). (2016). Evaluación de la sostenibilidad económica de la producción de café. Londres, Reino Unido.

Pereira, E., y Parrales, C. (2006). Evaluación de tres sistemas de manejo agronómico sobre el crecimiento, estructura productiva, acumulación de biomasa, rendimiento y calidad del café (Coffea arabica L.) vr. Costa Rica 95. Tesis de pregrado. Universidad Nacional Agraria. Managua, Nicaragua. Disponible en: https://repositorio.una.edu.ni/2002/1/tnf01p436.pdf

Sáenz, M., y De La Llana, A. (1990). Entomología sistemática. Managua, Nicaragua: UNA.

Siu, S. (2018). Uso de Beauveria bassiana (Bals y Vuils), equipos de aplicación y prácticas culturales para el manejo de broca del café (Hypothenemus hampei, Ferrari), en el cultivo del café (Coffea arabica L.), en Jalapa, Nueva Segovia, 2016-2017. Tesis de posgrado. Universidad Nacional Agraria. Managua, Nicaragua. Disponible en: https://repositorio.una.edu.ni/3770/1/tnh10s623.pdf

Vinces, D. (2018). Control químico del Pulgón negro de los citricos (Toxoptera aurantii) en Limonero (Citrus aurantífolia swingle) de Cieneguillo Norte, Sullana - Piura. Tesis de pregrado. Universidad Nacional de Piura. Piura, Perú. Diponible en : http://repositorio. unp.edu.pe/bitstream/handle/UNP/1297/AGR-VIN-OLE-18.pdf?sequence=1\&isAllowed=y

Williamson S., y Leheup, P. (2012). El uso de endosulfán se convierte en una Práctica Inaceptable. Londres, Reino Unido. 